\title{
Pial synangiosis in patients with moyamoya syndrome and sickle cell anemia: perioperative management and surgical outcome
}

\author{
Edward R. Smith, M.D., ${ }^{1}$ Craig D. McClain, M.D., ${ }^{2}$ Matthew Heeney, M.D., ${ }^{3}$ \\ and R. Michael Scott, M.D. ${ }^{1}$ \\ Departments of ${ }^{1}$ Neurosurgery, ${ }^{2}$ Anesthesiology, and ${ }^{3}$ Hematology/Oncology, Children's Hospital Boston, \\ Harvard Medical School, Boston, Massachusetts
}

\begin{abstract}
Object. Many children with sickle cell anemia (SCA) also have clinical and radiographic findings of an arteriopathy suggestive of moyamoya syndrome. These patients may continue to experience strokes despite optimal medical management. The authors wished to define features of moyamoya syndrome associated with SCA and determine the results of surgical revascularization in these patients at early and late follow-up.

Methods. The authors reviewed the clinical and radiographic records of all patients with moyamoya syndrome and SCA who underwent cerebral revascularization surgery using a standardized surgical procedure - pial synangiosis-from 1985 to 2008.

Results. Twelve patients had SCA and moyamoya syndrome. Six patients were female and 6 were male. The average patient age at surgery was 11.3 years (range 3-22 years). All patients presented with ischemic symptoms, 11 $(92 \%)$ with previous transient ischemic attacks, and $10(83 \%)$ with completed strokes. Eleven patients $(92 \%)$ had radiographic evidence of previous stroke at presentation. None presented with hemorrhage. Surgical treatment included pial synangiosis in all patients. Complications included 1 perioperative stroke, 1 wound infection, and 1 perioperative pneumonia. The average length of hospital stay was 5.7 days (including a 24-hour preoperative admission for hydration) and average blood loss was $92.5 \mathrm{ml} / \mathrm{hemisphere} \mathrm{(in} \mathrm{a} \mathrm{total} \mathrm{of} 19$ hemispheres). Clinical and radiographic follow-up with an average of 49 months (range 9-144 months) demonstrated no worsening in neurological status in any patient. No clinical or radiographic evidence of new infarcts was observed in any patient at late follow-up, despite disease progression in $13(68 \%)$ of 19 hemispheres.

Conclusions. The clinical and radiographic features of moyamoya syndrome associated with SCA appear comparable to primary moyamoya disease. Successful treatment of these patients requires multidisciplinary care involving hematologists, anesthesiologists, and neurosurgeons. Operative treatment of moyamoya syndrome using pial synangiosis appears to be safe and confers long-lasting protection against further stroke in this population, and provides an alternative for failure of optimal medical therapy in patients. This study underscores the potential merit of screening patients with SCA for moyamoya syndrome. (DOI: 10.3171.2009.01.FOCUS08307)
\end{abstract}

KeY Words • moyamoya • sickle cell anemia • stroke • pial synangiosis

$\mathrm{M}$ OYAMOYA syndrome is a chronic occlusive cerebrovascular disorder of unknown origin characterized by progressive stenosis of the bilateral supraclinoid ICAs with concomitant formation of tortuous arterial collaterals at the base of the brain, which reconstitute distal branches of the cerebral circulation. ${ }^{23,25}$ Current nomenclature distinguishes between moyamoya disease-the idiopathic presentation of angiographic moyamoya changes in both ICAs-and moyamoya

\footnotetext{
Abbreviations used in this paper: $\mathrm{ADL}=$ activities of daily living; ICA = internal carotid artery; $\mathrm{SCA}=$ sickle cell anemia; TCD = transcranial Doppler; TIA $=$ transient ischemic attack.
}

syndrome, the presentation of angiographic moyamoya changes found in association with a known pathological state (neurofibromatosis, Down syndrome, cranial irradiation, and others).

Recent reports have identified the presence of an arteriopathy in patients with SCA similar to moyamoya syndrome. ${ }^{8,10,19}$ The origin of this arteriopathy in patients with SCA is unclear, although hypotheses include repeated vascular injury from sickling, abnormal regulation of vasoconstriction, and intimal hyperplasia. ${ }^{5,9,15,17,20}$ Importantly, evidence suggests that the presence of this arteriopathy is common among patients with SCA and its presence is associated with an increased incidence of stroke., ${ }^{4,17}$ Approximately 100,000 patients have SCA in 
TABLE 1: Clinical and radiographic presentation data of the 12 patients in this series

\begin{tabular}{lc}
\hline \multicolumn{1}{c}{ Presentation } & No. of Patients (\%) \\
\hline clinical & \\
TIA & $11 / 12(92)$ \\
stroke (neurological deficit $>24$ hrs) & $10 / 12(83)$ \\
persistent neurological deficit from stroke & $7 / 12(58)$ \\
$\quad$ (deficit still evident at time of surgery) & \\
history of seizure & $3 / 12(25)$ \\
hemorrhage & $0 / 12(0)$ \\
transfusion regimen & \\
no. of patients on transfusion regimen & $12 / 12(100)$ \\
no. of patients w/new stroke while on trans- & $7 / 12(58)$ \\
fusion regimen & \\
radiographic presentation (12 patients/19 & \\
affected hemispheres) & \\
MRI & $12 / 12$ patients \\
& $(100) ; 19 / 19$ \\
& hemispheres \\
& $(100)$ \\
digital subtraction angiography & $9 / 12$ patients $(75) ;$ \\
& $13 / 19$ hemi- \\
mean Suzuki grade & spheres $(68)$ \\
stroke & 3.6 (range $3-5)$ \\
& $11 / 12$ patients $(92) ;$ \\
unilateral disease & $15 / 19$ hemi- \\
& spheres $(79)$ \\
\hline & $5 / 12$ patients $(42) ;$ \\
& 2 right, 3 left \\
\hline
\end{tabular}

the US, with nearly 1 in 10 children with SCA suffering a stroke before age $20 . .^{11,17}$

Currently, medical therapy for stroke reduction in patients with SCA is predominantly predicated on chronic transfusions, with patient selection derived from screening using TCD ultrasonography. Patients with elevated TCD velocities (> $200 \mathrm{~cm} / \mathrm{second}$ ) or a history of previous stroke are started on a regimen of transfusions. ${ }^{2,3,17}$ Those patients who respond to therapy enjoy a 10 -fold reduction in stroke risk. However, these transfusions must be continued indefinitely to maintain this protection. ${ }^{1,3,17}$ This protection comes with a cost, as there are risks associated with chronic transfusion, including infection, immune responses, and iron overload.

Importantly, not all patients respond to this therapy. As many as $43 \%$ of patients with SCA and strokes will have "moyamoya-like" collaterals on imaging studies, and patients with these findings have a 5-fold increased risk for recurrent stroke compared with patients with SCA without these collaterals.,11,17 These data suggest that there may be thousands of patients with SCA in whom medical treatment will fail and who will suffer repeated strokes despite optimal medical therapy.

Despite these statistics, there has been little impetus to consider surgical therapy in these patients. Consequently, the response of this group to surgical treatment is not well known. A small number of case series, including data from our institution, suggests that cerebral revascularization may be safe and confer long-lasting protection from stroke in this population. ${ }^{8,10,17,19}$ Although neurosurgeons are often familiar with the benefits of surgical management of moyamoya, hematologists - often the primary physicians of these complex patients with SCAare frequently reluctant to consider operative intervention in their patients. This study was undertaken to add to the growing body of literature describing the experience of surgically treating patients with SCA and moyamoya syndrome. Our patient series is equal in size to the largest series published to date and provides longer follow-up and improved radiographic documentation of the effects of surgery. We also document our experience successfully weaning a patient with SCA and moyamoya syndrome off of transfusion therapy following surgery.

\section{Methods}

We reviewed a consecutive surgical series of patients with moyamoya syndrome who underwent pial synangiosis from 1985 to 2008 to identify all patients who presented with moyamoya syndrome and SCA. In accordance with a protocol approved by an Institutional Review Board, the medical charts of these patients were retrospectively reviewed to determine patient age and sex at presentation, comorbid conditions, symptoms, results of radiographic studies (including CT, MR imaging/angiography, and cerebral arteriography), perioperative and late complications, length of follow-up, and long-term clinical and radiographic outcomes.

\section{Results}

\section{Patient Demographics}

Of all the patients with moyamoya syndrome during the period of study, 12 presented with SCA. There were 6 female and 6 male patients. The average age at presentation was 11.3 years, with a range of 3 to 22 years. Eleven of the patients were African American, and one was Hispanic.

\section{Clinical Presentation}

All 12 patients $(100 \%)$ presented with symptoms consistent with chronic cerebral ischemia, with a history of TIAs in 11 patients (92\%), and evidence of completed strokes in $10(83 \%)$. At the time of surgery, 7 patients (58\%) had persistent neurological deficits. Additionally, a history of seizures was present in 3 patients (25\%). No patients presented with hemorrhage (Table 1).

\section{Transfusion Regimen}

All 12 patients (100\%) were undergoing transfusions at the time of surgery. Of these, 7 (58\%) experienced a new stroke while on a transfusion regimen, indicating a failure of medical therapy (Table 1).

\section{Radiographic Presentation and Features}

In the 12 patients there was a total of 19 affected 


\section{Pial synangiosis for moyamoya in sickle cell anemia}

TABLE 2: Timeline for perioperative management of patients with moyamoya syndrome and SCA

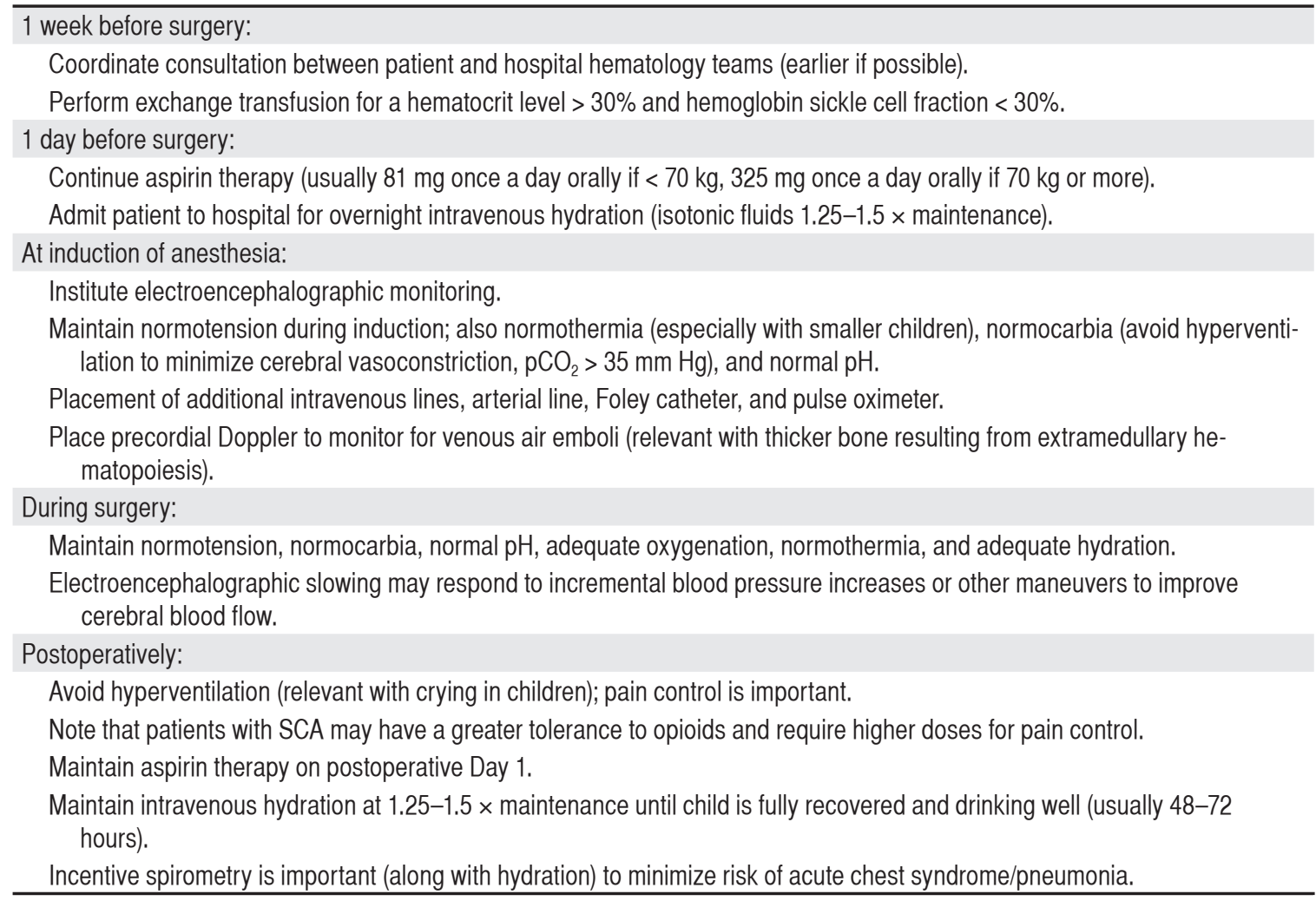

hemispheres. Preoperative MR imaging was available for all 12 patients and all 19 hemispheres. Digital subtraction angiography was available in 9 patients (75\%), involving 13 affected hemispheres $(68 \%)$. The mean Suzuki grade (a severity of arteriographic disease scale ranging from Stages I to VI, with VI being the most severe) ${ }^{25}$ at presentation on the affected side was 3.6, with a range of 3-5.

The angiographic characteristics of moyamoya syndrome in this patient population with SCA were indistinguishable from our series of over 300 moyamoya patients without SCA. Of the 12 patients, $11(92 \%)$ demonstrated the presence of previous infarction in 15 hemispheres on preoperative MR imaging (79\%). Only 1 patient had no radiographic evidence of previous stroke. Bilateral disease was present in 7 patients $(58 \%)$, and 5 patients $(42 \%)$ had only 1 side affected at the time of surgery. In the unilateral group, 3 affected hemispheres were on the left and the other 2 were on the right (Table 1).

\section{Operative Treatment}

All patients in this series underwent a standardized indirect cerebral revascularization procedure-pial synangiosis - to treat the affected hemisphere. ${ }^{23}$ Two of the patients with bilateral disease in this study were treated with staged operations with a 1-month interval between procedures. However, our current practice is to perform the operation on both sides in bilateral cases during a single anesthesia procedure (assuming intraoperative stability), and the subsequent 5 cases were so treated. The anesthesia and perioperative management of all patients with moyamoya is standardized at our institution. ${ }^{24}$ The additional presence of SCA in these complex patients has added several steps to our protocol, as outlined in Table 2.

\section{Surgical Results}

A total of 19 hemispheres were treated during 14 operations. There were 5 unilateral and 7 bilateral cases. The first 2 bilateral cases were staged, with the second side addressed 1 month later, while the subsequent 5 bilateral cases were completed during a single anesthesia procedure. Mean blood loss was $92.5 \mathrm{ml} /$ hemisphere (range 25-200 ml). Mean length of stay was 5.7 days (range 4-7 days), including a scheduled admission to the hospital on the night prior to surgery for preoperative hydration (Table 3).

\section{Perioperative Complications}

Perioperative complications occurred in 3 different patients, including 1 stroke, 1 wound infection, and 1 case of pneumonia/acute chest syndrome. The stroke occurred within the first 24 hours postoperatively and consisted of an expressive aphasia and mild right-sided weakness. Magnetic resonance imaging confirmed a small infarct in the left frontal lobe, and the patient experienced a substantial recovery within 72 hours. He was discharged home and had fully recovered by his 1-month postoperative visit.

The infection occurred in the setting of a child scratching open her incision after discharge. Abundant 
TABLE 3: Operative data for patients in this series

\begin{tabular}{lc}
\hline \multicolumn{1}{c}{ Parameter } & Value \\
\hline total no. of operations* & 19 (14 w/ follow-up) \\
unilateral & 5 \\
bilateral & 7 (14 total hemispheres) \\
average blood loss per operation & $92.5 \mathrm{ml}$ (range $25-200 \mathrm{ml})$ \\
average length of stay (including preop & 5.7 days (range 4-7 days) \\
hydration) & \\
complications & \\
deaths & $0 / 19(0 \%)$ \\
strokes & $1 / 19(5.3 \%)$ \\
TIAs & $0 / 19(0 \%)$ \\
seizures & $0 / 19(0 \%)$ \\
infections & $1 / 19(5.3 \%)$ \\
sickle cell-related issues (pneumo- & $1 / 19(5.3 \%)$ \\
nia/acute chest syndrome) & \\
\hline
\end{tabular}

* Two patients underwent staged operations 1 month apart.

pus was identified at exploration and the bone flap was ultimately removed. She remains asymptomatic neurologically and the synangiosis was able to be preserved.

Following an uneventful surgery, 1 child developed pneumonia and symptoms suggestive of acute chest syndrome. He had no neurological sequelae, but required a 1-week stay in a rehabilitation facility for pain management and respiratory therapy. There were no perioperative seizures, TIAs, or deaths in this series (Table 3 ).

\section{Clinical Outcome After Hospital Discharge}

The average follow-up duration after surgery was 49 months (range 9-144 months; Table 4). Patients were evaluated for clinical deterioration, comparing the findings at their preoperative examinations to those at follow-up using a modified Rankin outcome scale $(0=$ no symptoms; 1 = minor symptoms not affecting lifestyle; 2 $=$ minor handicap but independent in $\mathrm{ADL} ; 3=$ requiring some help with ADL; 4 = requiring substantial help with ADL; 5 = totally dependent). Using this scale, 5 (42\%) of 12 patients had improved neurological function and 7 $(58 \%)$ of 12 were stable.

One interesting patient had presented with recurrent TIAs and stroke during transfusions. Following surgery, there was interest in weaning him from these transfusions in an attempt to abrogate this unusual effect. The patient was started on oral hydroxyurea and was able to be suc- cessfully weaned off of all transfusions within 9 months. He has now spent more than 1 year without transfusions and remains stroke-free.

\section{Radiographic Outcome After Hospital Discharge}

All patients had postoperative MR imaging and/or angiography studies for review. The average radiographic follow-up was 32 months (range 6-113 months) with an average of 2.8 studies per patient (range 1-5 studies). Magnetic resonance imaging was available in 11 patients (92\%) and angiograms were performed in 5 patients (42\%).

There were no new strokes in any studies following hospital discharge (as discussed in the complications, 1 patient had a perioperative stroke), despite radiographic evidence of moyamoya progression in $13(68 \%)$ of 19 hemispheres (Fig. 1). New surgical collateral vessels were identified in 17 (89\%) of 19 hemispheres (Figs. 2 and 3). In all patients in whom postoperative arteriographic imaging could be evaluated using the Matshushima scale, ${ }^{14}$ Grade A collaterals were observed in $57 \%$ and Grade B collaterals in $43 \%$ of studies (Fig. 2).

None of the 5 patients with unilateral disease have developed disease on the contralateral side, although it is worth noting that 2 of these patients are only within 2 years of surgery. Analysis of the number of confirmed strokes during the period of time that patients were observed clinically reveals a complete absence of new infarcts following hospital discharge after surgical treatment (Fig. 4). Before surgery, there was a total of 16 strokes during a combined 26.3 patient-years. Following discharge, there were no new strokes over a combined 38.3 patient-years.

\section{Discussion}

Management of the cerebrovascular sequelae of SCA remains challenging. Although the recent advent of widespread TCD screening and therapeutic transfusions has markedly reduced the risk of stroke in many patients with SCA, a substantial fraction of those treated-as many as $20 \%$-will experience failure of medical therapy. ${ }^{16,17}$ Of those who experience this treatment failure, nearly half will have radiographic findings suggestive of moyamoya syndrome. ${ }^{4,17}$ In our group, 7 patients (58\%) experienced a stroke while receiving optimal transfusion management. This finding mirrors data in the hematology literature that suggests that patients with SCA who have moyamoya-like vessels on MR imaging do not experience the protective effect other patients experience with transfusion. ${ }^{4,11,13,17}$

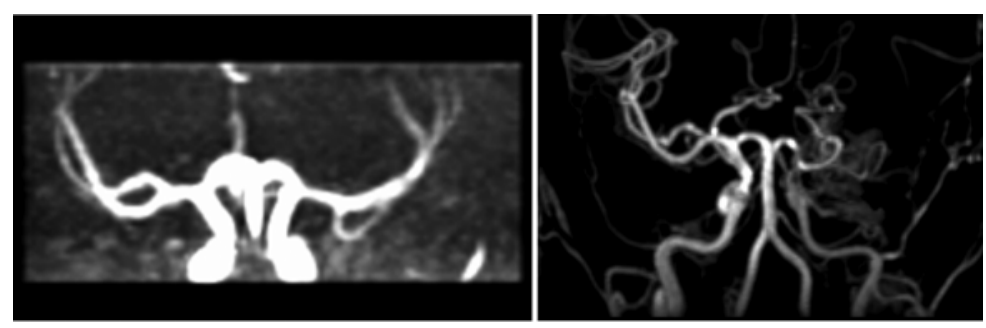

FIG. 1. Sequential MR angiograms (coronal views) documenting evidence of progressive moyamoya disease over a 5-year period. Note the diminution of the patient's left ICA with attendant development of moyamoya collaterals (right). 


\section{Pial synangiosis for moyamoya in sickle cell anemia}
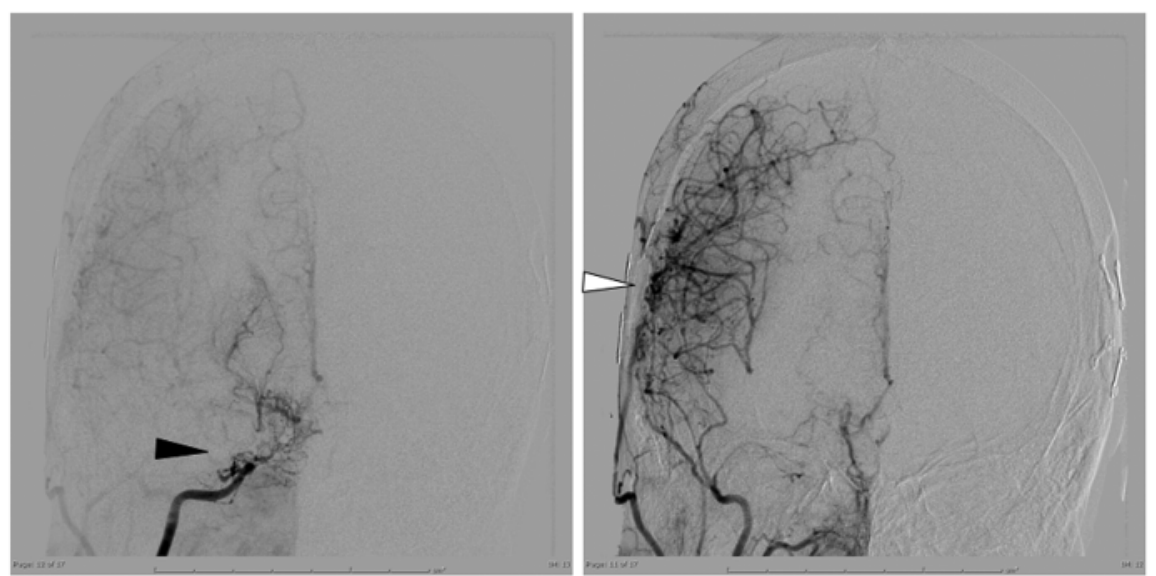

FIG. 2. Postoperative angiograms. Injection of the ICA reveals typical moyamoya changes (Suzuki Grade 5), including narrowing at the internal carotid apex and collateral vessels (black arrowhead). Injection of the external carotid artery reveals extensive revascularization at the surgical site (white arrowhead), providing supply throughout the hemisphere (Matsushima Grade A).

While acknowledging that transfusions are clearly beneficial for most patients with SCA, this identification of anatomical findings predictive of a treatment-refractory population compels physicians to search for alternative therapies. There is ample evidence that surgical therapyspecifically pial synangiosis-has low treatment morbidity ( $4 \%$ periprocedural risk of stroke) and confers near complete long-term abrogation of strokes. ${ }^{17,19,22,23}$ If patients with SCA have a moyamoya syndrome comparable to that of the "general" (non-SCA) moyamoya population, it follows that the benefits of pial synangiosis should be applicable as well.

Careful evaluation of the patients with SCA in our study revealed no major differences radiographically to non-SCA moyamoya cases. ${ }^{19}$ Ideally all patients with moyamoya are evaluated using angiography, given its ability to stage disease and identify collateral vessels so that they can be preserved at surgery. However, in contrast to other patients with moyamoya, patients with SCA have an increased risk of renal injury from dye loads and have the added risk of sickle crises (in addition to stroke) with general anesthesia, which is often required in children to tolerate catheter angiography. As a result of these factors, not all patients in this series were evaluated using angiography.

Clinically, the patients with SCA-moyamoya were also comparable, with the only appreciable difference being an older age at the time of surgery (11.3 years vs 7.1 years). ${ }^{19}$ Although biological factors may play a role, the fact that strokes are a known complication of SCA, and with an available treatment (transfusions) familiar to hematologists, it appears reasonable to assume that delayed referral to neurosurgery may be a major contributing factor to this discrepancy.

One other comparison in patients with non-SCA moyamoya exists in the analysis of individuals with unilateral moyamoya. None of the 5 patients in this series with unilateral disease developed progression on the contralateral side. Previous data have suggested that $30 \%$ of patients with unilateral findings will develop contralateral disease within an average of 2.2 years. ${ }^{22}$ As such, one might expect to see 1 or 2 of these 5 patients with some progression on the opposite site. However, it is worth noting that 2 of these unilateral patients are still within the 2.2-year period of follow-up. The limited number of unilateral patients in this series, together with the limited fol-
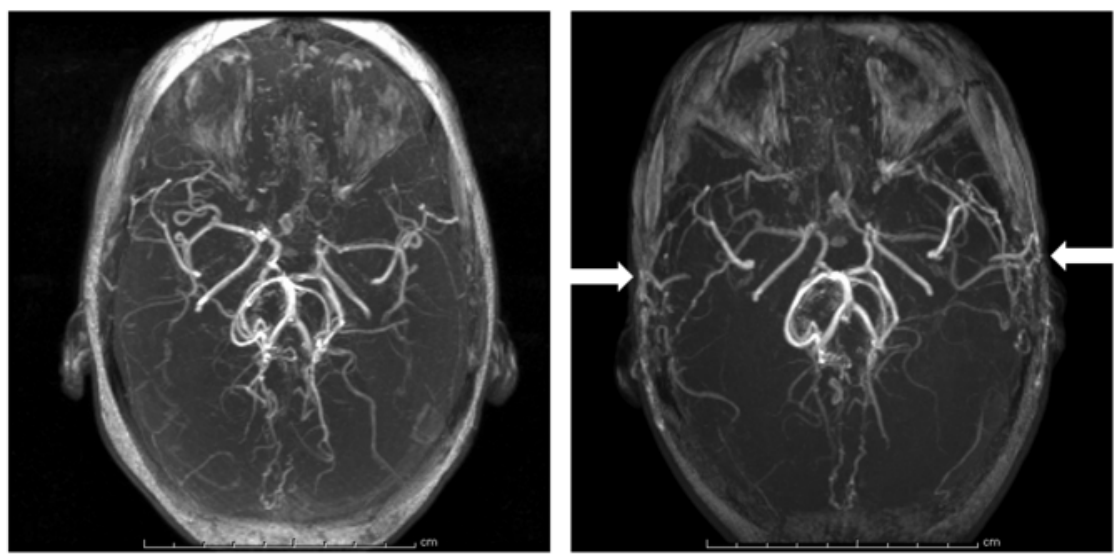

FIG. 3. Pre- (left) and postoperative (right) MR angiograms. Note the sites of pial synangiosis with hypertrophied donor arteries (superficial temporal arteries) and abundant collateral vessel development 1-year postoperatively (large arrows). 


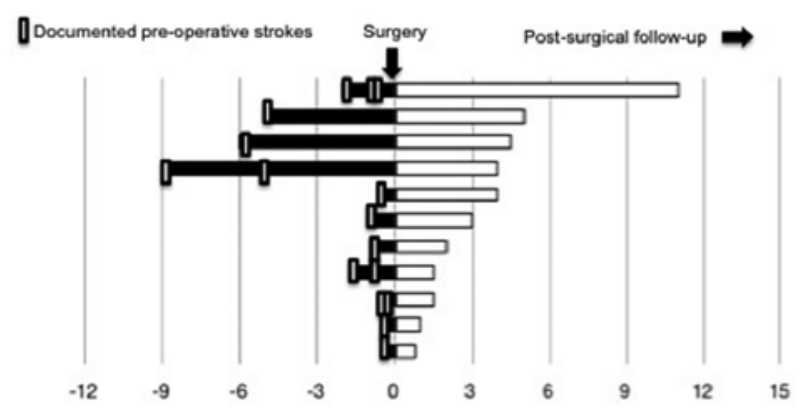

FIG. 4. Timeline depicting clinically and radiographically confirmed strokes in 11 patients from this series. One patient has not had any strokes. Note the abrogation of strokes (including those patients with multiple events) following surgery and during long-term follow-up.

low-up duration in 2 of these patients, makes it impossible to draw any definitive conclusions regarding this slight difference between the SCA-moyamoya population and the general moyamoya population.

There are increasing data to suggest that patients with SCA and moyamoya have favorable responses to surgery. ${ }^{8,10,17,19,26}$ These authors make note of the ease and success of "indirect" methods of cerebral revascularization, traits that are particularly important in this highrisk population. The patients in our series were treated exclusively using pial synangiosis. Our rationale for the use of this approach is derived from the ease of surgical technique, its applicability to all ages, and its documented safety and success in a wide range of moyamoya populations. $8,10,17,19,26$

Successful surgical management of moyamoya is dependent on safe anesthesia and perioperative care. We have gone to great lengths to provide coordinated multidisciplinary care of these patients. Involvement of experienced anesthesiologists and skilled hematologists is critical to the ability to achieve good outcomes. Detailed guidelines (from our institution and elsewhere) regarding the anesthetic issues surrounding both patients with SCA and individuals with moyamoya have been used in the construction of the protocol presented here (Table 2). $6,7,24$ As a component of caring for these complicated patients, consultation with hematology should be established prior to admission and maintained throughout the entirety of the patient's hospital course. ${ }^{21}$ We have found that admitting patients the night before a planned pial synangiosis for intravenous hydration smoothes the induction of anesthesia.

For patients with moyamoya, adequate postoperative analgesia is a primary goal, especially in small children in whom crying and hyperventilation may lead to cerebral vasoconstriction and consequent hypoperfusion. ${ }^{18}$ It should be noted that patients with SCA who have undergone frequent pain crises may be significantly tolerant to opioid analgesics and may require doses larger than usual to achieve analgesia. In addition, postoperative management should include a low threshold for administration of supplemental oxygen to prevent hypoxia, promote incentive spirometry, and encourage early mobilization.

Despite careful adherence to these guidelines, we found a total of 3 complications in this series of patients. It is difficult to identify specific causes of these problems, other than the obvious mechanical disruption of the surgical site, which appears to be the cause in the 1 wound infection in this series. The stroke rate of $5.3 \%$ per hemisphere is comparable to the $4 \%$ rate observed in the much larger series of all moyamoya patients undergoing identical treatment previously reported from our institution. ${ }^{19}$ Whereas the complication rates appear favorable in this series of patients with SCA-moyamoya and are comparable to patient series from other institutions, care must be taken in interpreting these results. ${ }^{10}$ The total number of patients in this study is small relative to non-SCA moyamoya series, emphasizing the need for increased reporting of institutional experiences with surgical treatment of these complex cases.

While acknowledging the limited number of patients reported here, this series nonetheless matches the size of the largest series to date (well-reported by Hankinson et al. ${ }^{10}$ ) while providing longer follow-up, improved postoperative radiographic documentation of surgical efficacy, and a description of an association between surgical therapy and successful cessation of transfusions. In this series, we demonstrate that surgical treatment of SCAmoyamoya with pial synangiosis is safe and provides long-lasting protection from ischemic symptoms.

With an average of just over 4 years of clinical follow-up, we found that all patients were clinically stable or improved following hospital discharge (as measured using a modified Rankin score), with no new strokes. These findings are complemented by extensive radiographic documentation substantiating the clinical data, with an average of approximately 1 imaging study (MR imaging and/or angiography) each year for nearly 3 years. Our protocol consists of an angiogram at 1-year postoperatively, followed by annual MR images. Angiograms were not possible in all patients due to travel issues or the aforementioned concerns regarding SCA, anesthesia, and dye load.

The stroke timeline (Fig. 4) allows the patients to be used as internal controls for comparison pre- and postsurgery. This complete abrogation of strokes-both clinically and confirmed radiographically-is particularly impressive when viewed in the context of continued progression of the moyamoya arteriopathy in $68 \%$ of affected hemispheres. Moreover, 7 of these patients (58\%) represent confirmed failures of medical therapy, and experienced strokes while undergoing transfusions. All of these patients showed evidence of moyamoya collaterals on preoperative imaging, documented using MR imaging in 3 patients and angiography in 4 patients. The cessation of events after surgery in this subset of patients underscores the potential effectiveness of surgical therapy in treating medically refractory individuals with SCA-moyamoya and stroke.

Even those patients who respond to medical therapy endure risks associated with frequent transfusions and, because the need for transfusions is chronic, experience increasing risk over time..$^{17}$ As highlighted by 1 of the patients in this series, the possibility of weaning patients off of transfusion therapy-and all its attendant long-term 
Pial synangiosis for moyamoya in sickle cell anemia

TABLE 4: Postoperative clinical and radiographic follow-up data

\begin{tabular}{|c|c|}
\hline Variable & Value \\
\hline mean follow up (mos) & 49 (range 9-144) \\
\hline \multicolumn{2}{|l|}{ clinical outcome following hospital discharge } \\
\hline strokes & $0 / 12(0 \%)$ \\
\hline TIA & $0 / 12(0 \%)$ \\
\hline new seizure disorder & $0 / 12(0 \%)$ \\
\hline headache & $4 / 12(33 \%)^{*}$ \\
\hline Rankin score change (preop vs follow-up) & $\begin{array}{l}5 / 12(42 \%) \text { improved } \\
7 / 12(58 \%) \text { stable } \\
0 / 12(0 \%) \text { worsened }\end{array}$ \\
\hline \multicolumn{2}{|l|}{ radiographic outcome following hospital discharge $\dagger$} \\
\hline average length of radiographic follow-up (mos) & 32 (range 6-113) \\
\hline average no. of postoperative images per patient & 2.8 (range 1-5) \\
\hline MRI imaging available & 11/12 (92\%), 17/19 hemispheres (89\%) \\
\hline angiogram available & 5/12 (42\%), 7/19 hemispheres (37\%) \\
\hline evidence of moyamoya progression & $13 / 19$ hemispheres $(68 \%)$ \\
\hline strokes following hospital discharge $\$$ & $0 / 17$ hemispheres $(0 \%)$ \\
\hline evidence of new surgical collaterals & $17 / 19$ hemispheres (89\%) \\
\hline \multicolumn{2}{|l|}{ Matsushima Grade } \\
\hline$A$ & 4/7 hemispheres (57\%) \\
\hline $\mathrm{B}$ & $3 / 7$ hemispheres $(43 \%)$ \\
\hline $\mathrm{C}$ & 0/7 hemispheres $(0 \%)$ \\
\hline \multicolumn{2}{|l|}{ stroke event rate before vs after surgery } \\
\hline stroke event rate before surgery & 16 strokes/26.3 patient-years \\
\hline stroke event rate following hospital discharge & 0 strokes/38.3 patient-years \\
\hline
\end{tabular}

risks - provides an additional benefit to surgical therapy beyond protection from cerebral ischemia.

In moyamoya syndrome, neurological status at the time of treatment predicts long-term outcome, suggesting that early diagnosis and treatment are important. ${ }^{17,19}$ Evidence supporting the presence of moyamoya in an at-risk population (patients with SCA and elevated TCD velocities), coupled with data supporting an effective treatment (surgical revascularization using pial synangiosis), could lead to better outcomes for these patients through earlier diagnosis and treatment. As such, we would advocate operating on patients with SCA and radiographic evidence of moyamoya and previous stroke, even in the absence of clinical symptoms. The findings presented in this patient series support the premise that patients with SCAmoyamoya are likely to experience failure of medical management, and could benefit from early detection from screening protocols when coupled with surgical revascularization, performed as soon as is feasible.

\section{Conclusions}

The clinical and radiographic features of moyamoya syndrome associated with SCA appear comparable to primary moyamoya disease. Although transfusion therapy is effective in preventing stroke for the majority of patients with SCA, a cohort of individuals with SCA-associated moyamoya appear to experience a high rate of unsuccessful standard medical management. However, treatment of SCA-associated moyamoya using pial synangiosis confers long-lasting protection against stroke. With long-term follow-up, all patients in this series remained clinically and radiographically stroke free after the initial perioperative period, despite evidence of radiographic progression of moyamoya in $68 \%$ of operated hemispheres. When coordinated with careful anesthetic and hematological management, revascularization surgery appears to be safe in these patients and appeared to be the major factor in preventing infarcts in the face of worsening stenosis of major cerebral arteries. This study suggests that screening patients with SCA for moyamoya may lead to improved neurological outcomes through earlier diagnosis of the disease and administration of pial synangiosis, an effective therapy.

\section{Disclaimer}

The authors report no conflict of interest concerning the materials or methods used in this study or the findings specified in this paper. 


\section{References}

1. Adams RJ, McKie VC, Brambilla D, Carl E, Gallagher D, Nichols FT, et al: Stroke prevention trial in sickle cell anemia. Control Clin Trials 19:110-129, 1998

2. Adams RJ, McKie VC, Carl EM, Nichols FT, Perry R, Brock $\mathrm{K}$, et al: Long-term stroke risk in children with sickle cell disease screened with transcranial Doppler. Ann Neurol 42:699-704, 1997

3. Adams RJ, McKie VC, Hsu L, Files B, Vichinsky E, Pegelow $\mathrm{C}$, et al: Prevention of a first stroke by transfusions in children with sickle cell anemia and abnormal results on transcranial Doppler ultrasonography. N Engl J Med 339:5-11, 1998

4. Dobson SR, Holden KR, Nietert PJ, Cure JK, Laver JH, Disco $\mathrm{D}$, et al: Moyamoya syndrome in childhood sickle cell disease: a predictive factor for recurrent cerebrovascular events. Blood 99:3144-3150, 2002

5. Drew JM, Scott JA, Chua GT: General case of the day. Moyamoya syndrome in a child with sickle cell disease. Radiographics 13:483-484, 1993

6. Firth PG: Anaesthesia for peculiar cells-a century of sickle cell disease. Br J Anaesth 95:287-299, 2005

7. Firth PG, Head CA: Sickle cell disease and anesthesia. Anesthesiology 101:766-785, 2004

8. Fryer RH, Anderson RC, Chiriboga CA, Feldstein NA: Sickle cell anemia with moyamoya disease: outcomes after EDAS procedure. Pediatr Neurol 29:124-130, 2003

9. Garza-Mercado R: Pseudomoyamoya in sickle cell anemia. Surg Neurol 18:425-431, 1982

10. Hankinson TC, Bohman LE, Heyer G, Licursi M, Ghatan S, Feldstein NA, et al: Surgical treatment of moyamoya syndrome in patients with sickle cell anemia: outcome following encephaloduroarteriosynangiosis. J Neurosurg Pediatrics 1:211-216, 2008

11. Kirkham FJ, DeBaun MR: Stroke in children with sickle cell disease. Curr Treat Options Neurol 6:357-375, 2004

12. Kirkham FJ, Hogan AM: Risk factors for arterial ischemic stroke in childhood. CNS Spectr 9:451-464, 2004

13. Lee BC, Park TS, Kaufman BA: MR angiography in pediatric neurological disorders. Pediatr Radiol 25:409-419, 1995

14. Matsushima T, Inoue T, Suzuki SO, Fujii K, Fukui M, Hasuo $\mathrm{K}$ : Surgical treatment of moyamoya disease in pediatric patients-comparison between the results of indirect and direct revascularization procedures. Neurosurgery 31:401-405, 1992
15. Merkel KH, Ginsberg PL, Parker JC Jr, Post MJ: Cerebrovascular disease in sickle cell anemia: a clinical, pathological and radiological correlation. Stroke 9:45-52, 1978

16. Pegelow CH, Adams RJ, McKie V, Abboud M, Berman B, Miller ST, et al: Risk of recurrent stroke in patients with sickle cell disease treated with erythrocyte transfusions. J Pediatr 126:896-899, 1995

17. Roach ES, Golomb MR, Adams R, Biller J, Daniels S, Deveber $\mathrm{G}$, et al: Management of stroke in infants and children: a scientific statement from a Special Writing Group of the American Heart Association Stroke Council and the Council on Cardiovascular Disease in the Young. Stroke 39:2644-2691, 2008

18. Sakamoto T, Kawaguchi M, Kurehara K, Kitaguchi K, Furuya $\mathrm{H}$, Karasawa J, et al: Postoperative neurological deterioration following the revascularization surgery in children with moyamoya disease. J Neurosurg Anesthesiol 10:37-41, 1998

19. Scott RM, Smith JL, Robertson RL, Madsen JR, Soriano SG, Rockoff MA: Long-term outcome in children with moyamoya syndrome after cranial revascularization by pial synangiosis. J Neurosurg 100:142-149, 2004

20. Seidman C, Kirkham F, Pavlakis S: Pediatric stroke: current developments. Curr Opin Pediatr 19:657-662, 2007

21. Shapiro ND, Poe MF: Sickle-cell disease: an anesthesiological problem. Anesthesiology 16:771-780, 1955

22. Smith ER, Scott RM: Progression of disease in unilateral moyamoya syndrome. Neurosurg Focus 24(2):E17, 2008

23. Smith ER, Scott RM: Surgical management of moyamoya syndrome. Skull Base 15:15-26, 2005

24. Soriano SG, Sethna NF, Scott RM: Anesthetic management of children with moyamoya syndrome. Anesth Analg 77:10661070, 1993

25. Suzuki J, Takaku A: Cerebrovascular "moyamoya" disease: disease showing abnormal net-like vessels in base of brain. Arch Neurol 20:288-299, 1969

26. Vernet O, Montes JL, O'Gorman AM, Baruchel S, Farmer JP: Encephaloduroarterio-synangiosis in a child with sickle cell anemia and moyamoya disease. Pediatr Neurol 14:226-230, 1996

Manuscript submitted December 19, 2008.

Accepted January 26, 2009.

Address correspondence to: Edward R. Smith, M.D., Harvard Medical School, Department of Neurosurgery, The Children's Hospital Boston, 300 Longwood Avenue, Boston, Massachusetts 02115. email: edward.smith@childrens.harvard.edu. 